\title{
Teses e dissertações da enfermagem brasileira sobre saúde da mulher no sistema prisional
}

\author{
Theses and dissertations of Brazilian nursing addressing women's health in the prison \\ system
}

Anderson Brito de Medeiros ${ }^{1}$, Lannuzya Veríssimo e Oliveira ${ }^{1}$, Glauber Weder dos Santos Silva ${ }^{1}$, Thaís Rosental Gabriel Lopes ${ }^{1}$, Jovanka Bittencourt Leite de Carvalho ${ }^{1}$, Francisco Arnoldo Nunes de Miranda ${ }^{1}$

\begin{abstract}
Objetivo: caracterizar a produção brasileira de teses e dissertações em enfermagem sobre a saúde de mulheres no sistema prisional. Métodos: estudo bibliométrico, a partir do panorama da produção científica, no âmbito da Pós-Graduação em Enfermagem brasileira, com coleta de dados no Catálogo de Teses e Dissertações da Coordenação de Aperfeiçoamento de Pessoal de Nível Superior. Resultados: 11 documentos fizeram parte da amostra. A maioria destes foi realizada durante mestrado $(63,6 \%)$, com predominância nas Regiões Nordeste $(63,6 \%)$, Sudeste $(18,1 \%)$ e Centro-oeste $(18,1 \%)$. Quanto às temáticas mais exploradas, as relações de mulheres/mães com os filhos apresentaram maior proporção (54,5\%). Predominaram os estudos qualitativos $(45,4 \%)$, com desenhos descritivos (36,3\%). Conclusão: teses e dissertações brasileiras de enfermagem sobre a saúde da mulher no sistema prisional constituíram-se por estudos qualitativos, provenientes das regiões Nordeste, Sudeste e Centro-oeste, as quais apresentaram lacuna na produção do conhecimento de enfermagem. Descritores: Enfermagem; Prisões; Prisioneiros; Saúde da Mulher; Bibliometria.
\end{abstract}

Objective: to characterize the Brazilian production of nursing theses and dissertations on women's health in the prison system. Methods: bibliometric study based on the panorama of scientific production in the context of Nursing post-graduations in Brazil. Data were collected in the Directory of Theses and Dissertations of the Coordination for Improvement of Higher Education Personnel. Results: eleven documents were included in the sample. Most of them were carried out during masters courses (63.6\%), with predominance of studies conducted in the Northeast (63.6\%), Southeast (18.1\%) and Midwest (18.1\%). Regarding the most explored themes, the relationship of women/mothers with their children presented a higher proportion (54.5\%). Qualitative studies (45.4\%), with descriptive designs (36.3\%) predominated. Conclusion: Brazilian nursing theses and dissertations on women's health in the prison system consisted of qualitative studies developed in the Northeast, Southeast and Midwest, and presented a gap in the production of nursing knowledge.

Descriptors: Nursing; Prisons; Prisoners; Women's Health; Bibliometrics.

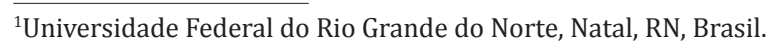




\section{Introdução}

No Brasil, de acordo com dados do Sistema Integrado de Informações Penitenciárias, em junho de 2016, a população carcerária feminina era de 42.355 mulheres. No entanto, ainda que esse número pareça insignificante, mediante a criminalidade geral, entre 2000 e 2016, a taxa de mulheres no sistema prisional aumentou em $455,0 \%{ }^{(1)}$. Essas mulheres tendem a ser jovens, afrodescendentes, mães, sem escolaridade, usuárias de drogas, características capazes de fomentar o ciclo de exclusão, antes mesmo da inserção no sistema prisional ${ }^{(2)}$.

Acrescente-se ao contexto dois agravantes: primeiro, as condições do cárcere, como superlotação, condições sanitárias e de saúde, jurídica, educacional e profissional; segundo, o fato de as prisões surgirem a partir de racionalidade que reconhecia a periculosidade como característica masculina, portanto, sendo as mesmas projetadas para estes, corroborando com ambiente prisional inadequado para abrigar, disciplinar e ressocializar ${ }^{(1)}$.

O encarceramento feminino é a privação da liberdade da mulher à sociedade, sobretudo, aos laços familiares. Características que podem levá-las ao adoecimento mental e físico ${ }^{(3)}$. Sabe-se que a assistência à saúde da mulher no sistema prisional deve ser assegurada por equipe multidisciplinar, ofertadas pelas Equipes de Saúde Prisional, bem como da Rede de Atenção à Saúde ${ }^{(4)}$. Dentre os profissionais, destaca-se a enfermagem, visto que o processo de trabalho desta possibilita maior permanência junto àquele que é assistido. Todavia, ainda que exista tal aparato legal, há lacuna entre o que é preconizado pela legislação, como normatizado pelos manuais ministeriais, e a efetiva garantia da assistência de enfermagem, tanto por carências estruturais ${ }^{(3-4)}$, quanto pelo déficit de informações.

No que se refere às pesquisas de enfermagem sobre a saúde do sistema prisional feminino, há um direcionamento para a saúde da mulher no ciclo grávido-puerperal ${ }^{(5)}$ ou relacionadas às Infecções Sexual- mente Transmissíveis (IST) ${ }^{(6)}$. Nessa perspectiva, faz-se necessário descrever a produção de conhecimento da enfermagem no contexto prisional, tendo em vista as especificidades da prisão e as necessidades de saúde das mulheres no cenário, para conhecer o que é produzido e suas lacunas, no intuito de contemplar maiores peculiaridades. Assim, analisar a produção da pós-graduação nos permite entender o panorama científico sobre a conjuntura.

É, nesse contexto, que se apresenta a questão de pesquisa do estudo: quais as características metodológicas e as tendências temáticas de dissertações e teses da enfermagem que abordam a saúde de mulheres no sistema prisional brasileiro? Portanto, objetivou-se caracterizar a produção brasileira de teses e dissertações em enfermagem sobre a saúde de mulheres no sistema prisional.

\section{Métodos}

Estudo bibliométrico, usando-se de técnicas quantitativas e qualitativas com análise de texto, a partir da produção científica de enfermagem, oriunda das pesquisas da Pós-Graduação em Enfermagem brasileira sobre saúde de mulheres no sistema prisional. A bibliometria constitui parte do percurso metodológico da pesquisa, ao analisar, quantativamente, a produção acadêmica das pesquisas e caracterizar o estado da arte sobre o tema ${ }^{(7)}$.

A escolha pelos relatórios das pesquisas da Pós-Graduação em Enfermagem para compor a presente pesquisa se deu pelo quantitativo da produção cientifica dentro dos programas stricto sensu, além de possibilitar as descrições dos estudos, quando comparados com artigos através do aprofundamento teórico, caminho metodológico, locais e instrumentos de coleta de dados detalhados. Os relatórios não têm barreiras de limitação de espaço, palavras e detalhes, julgados importantes para traçar melhor o panorama do contexto cientifico estudado.

O levantamento bibliográfico ${ }^{(8)}$ ocorreu em agosto de 2018, realizado por três pesquisadores in- 
dependentes que validaram a amostra final. Coletaram-se os dados no Catálogo de Teses e Dissertações da Coordenação de Aperfeiçoamento de Pessoal de Nível Superior, via Plataforma Sucupira, por meio de duas buscas isoladas, sem cruzamento nem uso de booleanos, com os descritores indexados na Biblioteca Virtual em Saúde: "prisões" e "prisioneiros". Utilizaram-se dos filtros "Mestrado", "Mestrado profissional", "Doutorado", "Doutorado Profissional" e "Área Avaliação - Enfermagem". Inicialmente, a avaliação ocorreu por leitura dos títulos e resumos.

Elencaram-se os critérios de elegibilidade: inclusão - (CI1) dissertações e teses de Programas de Pós-graduação em Enfermagem no Brasil e (CI2) que abordasse a saúde de mulheres no sistema prisional brasileiro. Excluíram-se: (CE1) dissertações e teses não disponibilizadas na íntegra nos bancos de dados eletrônicos de origem ou repositórios institucionais ou ausência de resposta dos autores ao pedido, via correio eletrônico, para acesso em completude ao relatório de pesquisa não encontrado eletronicamente.

Após o compilado de estudos selecionados, fez-se necessária leitura em totalidade destes para avaliação final do conteúdo. Seguiu-se a extração das variáveis: formação do autor; nível acadêmico; instituição de ensino; local de desenvolvimento do estudo; ano de defesa; área de conhecimento da enfermagem; referencial teórico utilizado; abordagem e desenho do estudo; temática estudada; técnicas de coleta de dados; e descritor de indexação.

Os dados foram organizados em planilha do software Microsoft Office Excel ${ }^{\circledR} 2016$ e submetidos à análise estatística descritiva, com apresentação dos valores em frequências absolutas e relativas. 0 material textual, oriundo dos resultados e das conclusões informados nos resumos dos relatórios de pesquisa selecionados, foi copiado/transcrito para documento Writer do software LibreOffice 6.1, para ser processado no Interface de R pour les Analyses Multidimensionnelles de Textes et de Questionnaires 0.7 Alpha 2 (Iramuteq), software que possibilita análise estatística textual, realizando agrupamento semântico do vocabulário ${ }^{(9-10)}$.

Realizou-se análise a partir do agrupamento de textos, em único arquivo, denominado de corpus, o qual foi submetido à análise e divisão por classificação hierárquica descendente. Como critérios de inclusão, foi realizada a associação das classes: frequência maior que o dobro da média de ocorrências no corpus com classe de valor khi2 igual ou superior a 3,84. Salienta-se que este valor é definido segundo grau de liberdade de 1 e significância de 95\%. Após processamento, realizou-se conjunto das classes semânticas por análise de conteúdo, formalizando categorias temáticas com o auxílio da análise fatorial de correspondência ${ }^{(9-10)}$

Destaca-se que não foi necessária apreciação ética para esta pesquisa, por se trabalhar de estudos de domínio público.

\section{Resultados}

Das 3.298 dissertações e teses encontradas no Catálogo da Coordenação de Aperfeiçoamento de Pessoal de Nível Superior, a partir dos parâmetros adotados, após refinamento e análise, foram pré-selecionados $22(0,6 \%)$ estudos para comporem a amostra e leitura detalhada. Na sequência, com a leitura na íntegra e em profundidade, permaneceram 11 (0,3\%) trabalhos (Tabela 1). Os demais $11(0,3 \%)$ foram excluídos, pois não retratavam a saúde de mulheres no sistema prisional brasileiro.

Tabela 1 - Estudos do Catálogo da Coordenação de Aperfeiçoamento de Pessoal de Nível Superior, a partir dos critérios de buscas, inclusão e exclusão

\begin{tabular}{lccccc}
\hline \multirow{2}{*}{ Indexadores } & \multicolumn{4}{c}{ Critério de inclusão } & \multicolumn{2}{c}{ Critério de Leitura na } \\
\cline { 2 - 5 } & \multicolumn{1}{c}{$\mathbf{1}$} & $\mathbf{2}$ & exclusão & íntegra & \\
\cline { 2 - 5 } & $\mathbf{n}(\%)$ & $\mathbf{n}(\%)$ & $\mathbf{n}(\%)$ & $\mathbf{n}(\%)$ & $\mathbf{n}(\%)$ \\
\hline Prisão & $1.997(60,6)$ & $17(0,5)$ & $2(0,1)$ & $22(0,6)$ & $8(0,2)$ \\
Prisioneiros & $1.301(39,4)$ & $7(0,2)$ & - & - & $3(0,1)$ \\
Total de relatórios & $3.298(100,0)$ & $24(0,7)$ & $2(0,1)$ & $22(0,6)$ & $11(0,3)$ \\
\hline
\end{tabular}


A totalidade dos estudos encontrados correspondeu ao período temporal de seis anos, entre 2012 e 2017. Com relação aos descritores utilizados para indexação dos relatórios, identificaram-se 46 (100\%) tesauros, majoritariamente, "Prisões" 33 (72,7\%), "Enfermagem" 25 (54,5\%) e "Saúde da mulher" 21 $(45,4 \%)$. Destes, 45 (98\%) foram encontrados nos Descritores de Ciências da Saúde (DeCS).

Em relação ao nível acadêmico, a maioria dos estudos foi realizada durante mestrado $7(63,6 \%)$, com predominância nas Regiões Nordeste 7 (63,6\%), Sudeste $2(18,1 \%)$ e Centro-oeste 2 (18,1\%). A Universidade Federal da Paraíba contemplava a maioria dos estudos 2 (18,1\%) (Figura 1).

\begin{tabular}{|l|l|}
\hline Regiões do Brasil/Instituição & $\begin{array}{c}\text { Autores/Ano/Nível Acadê- } \\
\text { mico }\end{array}$ \\
\hline Nordeste & \\
\hline $\begin{array}{l}\text { Universidade Federal do Rio Gran- } \\
\text { de do Norte }\end{array}$ & Galvão MCB/2012/Mestrado \\
\hline $\begin{array}{l}\text { Universidade Federal de Pernam- } \\
\text { buco }\end{array}$ & Guerra MCGC/2013/Mestrado \\
\hline Universidade Estadual do Ceará & Sousa AAS/2015/Doutorado \\
\hline Universidade Federal de Alagoas & França AMB/2015/Mestrado \\
\hline Universidade Federal da Paraíba & $\begin{array}{l}\text { Trigueiro DRSG/2015/Douto- } \\
\text { rado }\end{array}$ \\
\hline Universidade de Pernambuco & Silva LRP/2016/Mestrado \\
\hline Universidade Federal da Paraíba & Pinho TAM/2016/Doutorado \\
\hline Centro-Oeste & Bezerra RCC/2013/Mestrado \\
\hline Universidade de Goiás & Silva ERPH/2017/Mestrado \\
\hline $\begin{array}{l}\text { Universidade Federal do Mato Gros- } \\
\text { so do Sul }\end{array}$ & Fochi MCS/2014/Mestrado \\
\hline Sudeste & Mariano GJS/2016/Doutorado \\
\hline Universidade Estadual de Campinas & \\
\hline Universidade de São Paulo &
\end{tabular}

Figura 1 - Caracterização das publicações, segundo unidade federativa, instituição, autores, ano, nível acadêmico $(n=11)$

Os estudos eram, predominantemente, qualitativos $5(45,4 \%)$, com predomínio dos desenhos de estudos descritivos $4(36,3 \%)$. No que tange ao referencial teórico dos estudos, a Teoria das Representações Sociais serviu de fundamentação teórica em 2 $(18,1 \%)$ dos relatórios e, em 2 (18,0\%) das pesquisas foi utilizado, o referencial teórico da enfermagem. No que diz respeito à técnica de coleta de dados, a entrevista correspondeu a $6(54,5 \%)$ (Tabela 2$)$.

Tabela 2 - Variáveis da abordagem metodológica, desenho do estudo, referencial teórico e técnica de coleta de dados

\begin{tabular}{lc}
\hline Variáveis & n (\%) \\
\hline Abordagem metodológica & \\
Qualitativa & $5(45,4)$ \\
Quantitativa & $3(27,2)$ \\
Métodos mistos & $3(27,2)$ \\
Desenho de estudo & \\
Descritivo & $4(36,3)$ \\
Transversal & $2(18,1)$ \\
Revisão integrativa & $1(9,1)$ \\
Fenomenologia & $1(9,1)$ \\
Teoria fundamentada nos dados & $1(9,1)$ \\
Censitário & $1(9,1)$ \\
Triangulação dos métodos & $1(9,1)$ \\
Referencial teórico & \\
Não esclarecido & $5(45,4)$ \\
Teoria das Representações Sociais & $2(18,1)$ \\
Teoria da Adaptação de Roy & $1(9,1)$ \\
Fenomenologia Social & $1(9,1)$ \\
Interacionismo Simbólico & $1(9,1)$ \\
Teoria do Torna-se Mãe & $1(9,1)$ \\
Técnica de coleta de dados & \\
Entrevista & $6(54,5)$ \\
Momento observacional & $3(27,2)$ \\
Entrevistas-genograma-ecomapa & $1(9,1)$ \\
Associação livre de palavras & $1(9,1)$ \\
\hline
\end{tabular}

Após o processamento do corpus realizado oriundos da transcrição dos 11 resumos (resultados/ conclusões), foi evidenciado a ocorrência de 2.480 palavras. Destas, 947 apresentaram-se de formas distintas (média de 2.62 para cada forma de palavra), critério este utilizado como inclusão dos elementos no dendograma. De forma sequencial, realizou-se a análise lexicográfica dos textos a partir do software, o qual realiza a partir de corte de 40 caracteres, correspondendo a análise de $54(73,97 \%)$ de 73 segmentos textuais. Portanto, considerou-se o aproveitamento na retenção satisfatório. 
Utilizou-se do método de Classificação Hierárquica Descendente e alcançaram-se seis classes, que agruparam as expressões e palavras usadas pelos autores e evidenciaram os conteúdos temáticos dos resumos presentes nos relatórios. Após análise de conteúdo, observou-se que as áreas temáticas predominantes elaboraram três categorias temáticas: as classes 5 e 6 formaram a categoria I - Situação de saúde de mulheres no sistema prisional, com retenção de $3(27,2 \%)$ dos estudos e compostas pelas seguintes palavras (valor de ${ }^{2}$ ): exame $6(38,81)$; alteração 5 $(31,68)$; realizar $5(8,91)$; resultado $6(6,62)$ e medicação $5(24,24)$; hábito $5(24,24)$; problema $7(14,92)$ e uso $5(13,8)$.

Relacionado à classe 2, formou-se a categoria II - Vivência sexual e HIV/Aids/IST (vírus da imunodeficiência humana/ Síndrome da imunodeficiência adquirida), no contexto de mulheres no sistema prisional, com $2(18,1 \%)$ da totalidade dos estudos, em que foram encontradas as palavras (valor $\mathrm{de}^{2}$ ): aids $6(38,81)$; sexo $6(25,11)$; sentimento $5(18,55)$; 8 sexual $(16,92) ;$ prático $8(16,92)$. Sobre as classes 1 e 3, emergiu a categoria III, denominada de Relações de mulheres/mães com filhos(as) no sistema prisional, apresentando quantitativo de $6(54,5 \%)$ dos relatórios, com as palavras (valor de ${ }^{2}$ ): mãe 7 $(14,92)$; mulher 19 (10,81); filho $10(8,06)$ e maternidade 7 (12,93); ambiente prisional 7 (6,7); e filho 10 $(6,64)$. Destaca-se que a classe 4 não obteve resultado estatístico satisfatório para inclusão de vocabulários na classe.

\section{Discussão}

Observou-se como limitação do estudo o pequeno número amostral do estudo a partir dos parâmetros da amostra realizada. Reconhece-se o acesso das produções contidas apenas no catálogo da Coordenação de Aperfeiçoamento de Pessoal de Nível Superior, o que pode ter limitado o acesso às produções de teses e dissertações em outros catálogos. No entanto, este estudo torna-se relevante ao descrever o estado da arte das pesquisas nacionais sobre saúde da mulher no sistema prisional, demonstrando as especificidades e o contexto da produção científica sobre a vivência dessas mulheres.

No entanto, a análise das dissertações e teses encontradas apontou para necessidade da exequibilidade do papel do enfermeiro no contexto prisional, sendo este um dos profissionais capaz de promover mudanças no cenário e contribuir diretamente no melhoramento da saúde dessas mulheres. Evidenciou-se que o tema encontra-se em ascensão no cenário da pesquisa brasileira. Tal afirmação também é fundamentada pela evidência no crescimento do interesse da academia nessa área de estudos, a partir dos anos 1990 e $2000^{(11)}$.

No cenário da pós-graduação em enfermagem, o número das pesquisas sobre a temática é ínfimo, compreendido entre 2012 e 2017, como se observou neste estudo bibliométrico. É possível que o pequeno número de estudos sobre a temática seja pela invisibilidade social do cárcere, refletida no contexto das disciplinas acadêmicas, incluindo a enfermagem ${ }^{(12)}$. A esse fato, somam-se os desafios da abordagem de conteúdos temáticos sobre cuidados às populações-chave e vulneráveis na formação em enfermagem. Assim, também se insere nesse contexto o ensino das condições e intervenções em saúde e enfermagem para mulheres em sistema prisional, defendido nas diretrizes curriculares de $2001^{(12)}$.

Assim, constata-se que o crescimento no tocante ao aprisionamento feminino não foi acompanhado pelo desenvolvimento da produção científica sobre tal temática. $\mathrm{O}$ aumento exponencial do número de cárcere feminino difere da lentidão sobre a produção científica ${ }^{(1,3)}$. Destaca-se a predominância dos estudos de enfermagem referentes às mulheres no sistema prisional na Região Nordeste do Brasil, representada em $63,0 \%$, apesar de não contemplar maior número de mulheres detentas nos presídios, com destaque para pesquisas no estado da Paraíba, diferentemente da Região Sudeste, a qual possui o maior contingente ${ }^{(13)}$. Referente ao percurso metodológico das pes- 
quisas encontradas na bibliometria houve predomínio de abordagem qualitativa, direcionados a compreender a vivência de mulheres em presídios. Certamente, no cenário prisional, as pesquisas qualitativas tornam-se mais exequíveis à aproximação do pesquisador com a mulher, uma vez que se faz necessário emergir no campo e conhecer minuciosamente a população. Ressalta-se ainda que as pesquisas de enfermagem são direcionadas, predominantemente, nas relações humanas e nas singularidades dos indivíduos, justapondo as pesquisas qualitativas ${ }^{(14)}$. Aponta-se, ainda, que dentro de contexto histórico, o aprisionamento em si tem sido prioritariamente estudado pelas ciências sociais e jurídicas, disciplinas que utilizam mais os métodos qualitativos ${ }^{(15)}$.

Dentre os referenciais teóricos observados, o mais recorrente foi a Teoria das Representações Sociais, a qual se inseriu. Nos estudos da enfermagem brasileira na década de 1990 e, nos dias atuais, tem apresentado ascensão, além do crescimento enquanto arcabouço teórico e metodológico. Este referencial oportuniza conhecer os significados, valores e as singularidades, os quais mulheres encarceradas vivenciam dentro do contexto prisional ${ }^{(16)}$.

Sabe-se que as teorias de enfermagem devem ser consideradas aplicáveis ao cenário, em virtude da importância desta na práxis, com direção ao cuidado do sujeito e da coletividade. Todavia, apenas um estudo se apropriou de abordagem teórica da enfermagem. Acrescenta-se que a saúde da mulher no sistema prisional se apresenta inclinada para modelos teóricos da enfermagem, como a Teoria das Necessidades Humanas Básicas de Wanda de Aguiar Horta e Teoria Ambientalista ${ }^{(17)}$. No entanto, apenas um estudo utilizou a Teoria de Adaptação de Callista Roy, o que confirma o distanciamento dos pesquisadores com as teorias de enfermagem.

Relativo à etapa de análise dos resultados dos estudos, emergiram-se três categorias que apresentaram o panorama temático da produção de enfermagem relacionada à saúde de mulheres no contexto prisional.
A primeira categoria dos resultados refere-se à Situação de saúde de mulheres no sistema prisional. 0 percentual significativo de mulheres no sistema prisional reflete, diretamente, sobre a importância de acompanhar as situações de vulnerabilidades e iniquidades com relação aos prejuízos à saúde que estas apresentam ${ }^{(5)}$. Estudos que contemplam esta categoria demonstram que o câncer do colo do útero; as afecções respiratórias, vasculares; e os problemas odontológicos são aspectos potencializados pela ausência de integralidade à saúde feminina, associados aos hábitos de vida, como uso de tabaco, drogas ilícitas e automedicação. Sob essas circunstâncias, que os problemas de saúde de mulheres neste cenário estão atrelados às condições inadequadas de saúde e hábitos sociais ${ }^{(18)}$.

No que concerne aos dados obstétricos e ginecológicos, esta pesquisa bibliométrica ratifica que mulheres no sistema prisional eram, em maioria, jovens, mães, com histórico de multiplicidade de parceiros sem proteção; relatos de abuso sexual na infância e no sistema prisional, os quais elevam os riscos de desenvolverem o câncer de colo do útero. Ademais, os estudos desta categoria apresentam dados relativos à citológica oncótica dessas mulheres dentro e fora dos sistemas prisionais, e com alterações em maioria ${ }^{(19)}$.

Compreende-se a importância dos achados ao corroborar com pesquisadores $^{(19)}$ que afirmam em estudos a predominância de mulheres jovens solteiras ou separadas, baixa escolaridade, provenientes de contexto de nível socioeconômico precário e escassez de acesso aos serviços de saúde antes de serem mantidas no sistema prisional.

Sinaliza-se, que essa população feminina possui contato maior com situações que inferem sobre a vulnerabilidade sexual; reprodutiva, psicossocial e afetiva, as quais estão intrinsicamente, relacionadas ao estilo de vida construído dentro do sistema. 0 ócio, o sedentarismo, as restrições alimentares e de lazer colaboram para o prejuízo da estabilidade física-emocional. É assertivo discorrer os impasses de promover educação em saúde e estabelecer rotinas que edifi- 
quem a qualidade de vida destas. Sob esta perspectiva, alerta-se que as condições de vida e saúde da população carcerária sofrem influências ambientais ${ }^{(5,20)}$.

Ao adentrar no sistema prisional, as mulheres tendenciam ao desenvolvimento de agravos à saúde, como: exposição ao HIV, IST, diabetes e hipertensão. Não obstante, é comum realidade aquém da indicada pelas normas ministeriais nos presídios femininos, com escassez de equipe de saúde multiprofissional que possa oferecer integralidade e equidade da assistência à saúde ${ }^{(21)}$. Soma-se a esses fatores, a ausência de locais apropriados para o atendimento a essas mulheres dentro do sistema prisional.

Diante da realidade desafiadora, na busca pela promoção e prevenção de doenças e respectivos agravos à saúde de mulheres no sistema prisional brasileiro, a Política Nacional de Atenção Integral à Saúde das Pessoas Privadas de Liberdade no Sistema Prisional elenca como um dos objetivos o direito à integralidade das ações de vigilância à saúde pautadas no Sistema único de Saúde ${ }^{(1)}$. Compreende-se que o aprisionamento favorece o reconhecimento dos determinantes e condicionantes de saúde, os quais oportunizam o direcionamento da assistência ao processo saúde-doen$\mathrm{ça}^{(21)}$.

Nesse contexto, no intuito da exequibilidade das ações programáticas na política de saúde, o enfermeiro, profissional com papel primordial no reconhecimento das particularidades desta população, deve captar, acolher, orientar e promover ações que propiciem atenuar os agravos à saúde dessas mulheres. Para tanto, é imprescindível enfermeiros sensibilizados e capacitados com a realidade pessoal de cada mulher, conferindo-as os direitos humanos básicos de cidadã, desprovidos de pré-julgamentos ${ }^{(22)}$.

Compreende-se que o enfermeiro possui papel fundamental, enquanto equipe interdisciplinar, na contribuição de ações integradas e voltadas à assistência de saúde de mulheres no sistema prisional. Desta forma, o estudo ${ }^{(22)}$ corrobora com papel do enfermeiro no controle de doenças inoportunas e comuns a essas mulheres, assim como o olhar ampliado às situações de saúde decorrentes dos hábitos e do estilo de vida dessas mulheres.

A segunda categoria, encontra-se intitulada Vivência sexual e HIV/Aids/IST no contexto de mulheres no sistema prisional. O HIV/Aids, atualmente, é considerado enfermidade que acomete os indivíduos e está intrinsecamente relacionado aos determinantes e condicionantes, no que tange aos indicadores epidemiológicos. Todavia, o sistema prisional se apresenta, de um lado, como protetor da sociedade frente à criminalidade; do outro, agentes das condições de promoção à saúde, o que, na realidade, torna-se agravante e complicador para mulher, no que diz respeito ao processo saúde-doença, devido à fragilidade no sistema prisional, assim como a ambiência insalubre e hostil que assola tais mulheres, juntamente com os altos riscos de doenças oportunizadas pela conjuntura ${ }^{(23)}$.

No contexto da mulher no sistema prisional, as mulheres se apresentam esclarecidas ${ }^{(24)}$ sobre as formas de contágio do HIV e a possível consequência de desenvolver a Aids. No entanto, apesar de conhecerem as formas de transmissão e descreveram aspectos emocionais e psicossociais ao lidarem com a doença, quais sejam: medo, solidão, rejeição e preconceito, as mulheres tendenciaram o pensamento às consequências físicas que a infecção desencadeia, além dos aspectos psíquicos que são afetados pela estigmatização social da doença.

Desse modo, aponta-se que o levantamento sorológico de detentas para detecção precoce de anticorpos positivos a doenças infecciosas é essencial para monitoramento e prevenção da disseminação do HIV e IST, em virtude da condição insalubre do sistema prisional. 0 manejo adequado de tais medidas preventivas é de responsabilidade dos profissionais de saúde, em especial de enfermeiros, que assistem as mulheres neste contexto, tendo em vista o temor acerca da Aids, relacionado ao percurso histórico que perpassam os indivíduos acometidos pelo HIV ${ }^{(24)}$.

Relativo às demais IST, como: sífilis, hepatites, herpes, gonorreia, trichomonas, pode-se afirmar que estas doenças são disseminadas pelas práticas sexuais 
inseguras dentro dos presídios, associado ao desconhecimento de condutas adequadas. Estas afecções comprometem o sistema imunológico de mulheres e, indiretamente, a saúde de companheiros nas visitas íntimas, bem como as relações homoafetivas estabelecidas dentro dos presídios ${ }^{(24)}$.

No que concerne à sexualidade, especificamente na visita íntima, questões de gênero sobrepõe o contexto, em razão da impraticabilidade dos direitos sexuais referentes às mulheres no sistema prisional e consentidas aos homens. As mulheres são vitimizadas por normatizações e burocracias divergentes dos homens, ao terem a praticabilidade da sexualidade restrita. Desta forma, demandas como sexualidade, cuidado em saúde, efetivação dos direitos femininos, bem com a garantia da visita íntima são desprezadas pelo sistema ${ }^{(25)}$.

Portanto, há de se considerar que as mulheres no sistema prisional são vulneráveis ao HIV/Aids e IST, as quais podem ser adquiridas por meio de práticas sexuais sem medidas preventivas, bem como o compartilhamento frequente de objetos íntimos ou de uso pessoal. Em decorrência das manifestações da sexualidade, torna-se salutar que os profissionais de saúde promovam a promoção e prevenção dos agravos da saúde, a partir do incentivo ao comportamento protetor das mulheres, por meio de ações educativas e programáticas, com vistas adequada à saúde sexual e reprodutiva ${ }^{(24-25)}$. Não obstante, este cuidado deve ser pautado na integralidade e singularidade do indivíduo.

Na perspectiva do modelo flexneriano do cuidado, com enfoque na saúde da mulher no sistema prisional, é perceptível, na conjuntura, identificar a fragilidade da assistência no processo saúde-doença. Indubitavelmente, a inexistência da educação e promoção à saúde no sistema prisional desencadeia a elasticidade das políticas públicas direcionadas ao cenário, o acarreta impraticabilidade dos direitos de mulheres em instituições prisionais, uma vez que estas desconhecem as garantias destes ${ }^{(25)}$.

A terceira categoria refere-se as Relações de mulheres/mães com filhos(as) no sistema prisional. As relações familiares tendem a ser fragilizadas quando a mulher se encontra no cenário prisional, em virtude do distanciamento de companheiros, filhos, parentes e pessoas de convívio. Este fato infere sobre o íntimo da mulher-mãe e, consequentemente, no protagonismo feminino e a visibilidade desta pela sociedade. Destaca-se, dentro do universo da mulher, o papel materno, quando esta precisa o exercer longe do contato diário com o(a) filho(a), o que fragiliza o vínculo de ambos. Esse distanciamento pode acarretar desfechos inóspitos para filhos(as), pois, na maioria das vezes, necessitam ser cuidados por familiares, amigos e, até mesmo, encaminhados a abrigos ou instituições públicas ${ }^{(26-28)}$.

Nesse sentido, pesquisas que se enquadram nessa categoria demonstram a importância da maternagem como estratégia de ressocialização e enquadramento da mulher "transviada" do padrão, socialmente, estabelecido e aceitável, bem como a dicotomia da mãe/presidiária com filho(a). Desta forma, este estudo destaca o cuidado materno ao(à) filho(a), o qual ocorre dentro de ambiente limitado e inapropriado, fragmentado à maternagem ${ }^{(26)}$.

Não obstante, as práticas maternas no sistema prisional referentes a esse cuidado perpassam pelo temor vivenciado no intramuro e, nesta lógica, estu$\mathrm{do}^{(26)}$ mostra as condições do encarceramento feminino como inapropriadas e hostil para díade. Ademais, a necessidade do aleitamento materno nesta conjuntura torna-se preocupante e desafiadora. Os significados atribuídos ao ato de amamentar, pelas mulheres no sistema prisional, estão intrinsecamente ligados à projeção do futuro, com a certeza do rompimento, com data marcada da díade ${ }^{(26,28)}$.-

Como extensão dos cuidados maternos, estudo ratifica sobre a fragilidade da assistência gravídico-puerperal, haja vista a carência de comprometimento e investimento das políticas de saúde estabelecidas a esse público ${ }^{(26)}$. Nesse mesmo ínterim, os cuidados à mulher/presidiária/mãe referente ao aleitamento materno surgem como mecanismo importantes da 
maternidade, àquelas que se encontram no período puerperal, na efetivação do vínculo mãe-filho ${ }^{(27-28)}$.

Assim, o fortalecimento das ações, no que tange ao papel da mulher-mãe pelos profissionais de saúde do sistema prisional, é primordial para minimizar o sofrimento materno, devido à obrigatoriedade da separação entre mãe e filho ${ }^{(26)}$. Considera-se, ainda, que o medo, a incerteza, ruptura, insegurança e culpa são características da mulher aprisionada que vivencia o papel materno no sistema prisional ${ }^{(27-29)}$.

A atenção direcionada à mulher no sistema prisional deve ser assegurada de forma igualitária, uma vez que a garantia dos direitos a essas mulheres, conforme a Constituição Federal de 1988 é para toda população brasileira, sem distinção de contexto. 0 estabelecimento das medidas justas para mulheres no sistema prisional foi elencado pelas Nações Unidas, segundo as regras de Bangkok, aprovadas em 2010, na perspectiva de melhoramento dos direitos voltados à família, à saúde feminina, sexual e reprodutiva, bem como dos(as) filhos(as) que vivenciam o encarceramento. Ademais, sabe-se que encarceramento per si manifesta-se como dificultador da promoção à saúde dessas mulheres e crianças, desde a concepção, igualmente do bem-estar biopsicossocial ${ }^{(29)}$.

\section{Conclusão}

Os estudos encontrados sobre a situação de saúde das mulheres dentro do sistema prisional voltaram-se para as vivências das mulheres com as infecções sexualmente transmissível, bem como para as relações construídas entre mãe e filho dentro do presidio. Evidenciou-se ainda, predominância dos estudos de abordagem qualitativa, desenvolvidos, principalmente, na Região do Nordeste do país. Ressalta-se, a pouca diversidade temática e metodológica nas pesquisas abordando a temática, o qual demonstra lacuna da produção do conhecimento de enfermagem, necessitando abordar outros aspectos a partir da realidade do sujeito.

\section{Colaborações}

Medeiros AB, Oliveira LV, Silva GWS e Lopes TRG contribuíram na concepção, projeto e interpretação dos dados; Redação do artigo, revisão crítica relevante do conteúdo intelectual e aprovação final da versão a ser publicada. Carvalho JBL e Miranda FAN colaboraram com análise e interpretação dos dados, redação do artigo, revisão crítica relevante do conteúdo intelectual e aprovação final da versão a ser publicada.

\section{Referências}

1. Ministério da Justiça (BR). Departamento de Execução Penal. Sistema Integrado de Informações Penitenciárias. Levantamento Nacional de Informações Penitenciárias INFOPEN Mulheres. Brasília: Ministério da Justiça; 2017.

2. Pereira EL. Families of incarcerated women, health promotion and access to social policies in the Federal District, Brazil. Ciênc Saúde Coletiva. 2016; 21(7):2123-34. doi: http://dx.doi. org/10.1590/1413-81232015217.16792015

3. Diuana V, Marilena CDV, Ventura M. Women in Brazilian prisons: tensions between punitive disciplinary order and maternity prescriptions. Physis. 2017; 27(3):727-47. doi: http://dx.doi. org//10.1590/S0103-73312017000300018

4. Lermen HS, Gil BL, Cúnico SD, Jesus LO. Health in prison: analysis of health social policies targeting the Brazilian prison population. Physis. 2015; 25(3):905-24. doi: http://dx.doi.org/10.1590/ s0103-73312015000300012

5. Mustofa M, West B, Supadmi MS, Sari H. Challenges to mothering while incarcerated: preliminary study of two women's prisons in Java, Indonesia. Int J Prisoner Health. 2019; 15(1):37-45. doi: http://dx.doi.org/10.1108/IJPH-06-2017-00316

6. Pinho TAM, Silva AO, Pimenta, CJL, Moreira MASP. Social representations of imprisoned women on Acquired Immunodeficiency Syndrome. Rev Rene. 2018; 19e3280. doi: http://dx.doi. org/10.15253/2175-6783.2018193280 
7. Quevedo-Silva F, Santos EBA, Brandão MM, Vils L. Bibliometric Study: guidelines on its application. Rev Bras Mark. 2016; 15(2):246-62. doi: http:// dx.doi.org/10.5585/remark.v15i2.3274

8. Machado Junior C, Souza MTS, Parisotto IRS, Palmisano A. As leis da bibliometria em diferentes bases de dados científicos. Rev Ciênc Adm. 2016; 1(1):111-23. doi: http://dx.doi. org/10.5007/2175-8077.2016v18n44p111

9. Marchand P, Ratinaud P. L'analyse de similitude appliqueé aux corpus textuels: les primaries socialistes pour l'election présidentielle française (septembre-octobre 2011) [Internet]. 2012 [cited July 25, 2019]. Available from: http://lexicometrica.univ-paris3.fr/jadt/jadt2012/Communications/Marchand,\%20Pascal\%20 et\%20al.\%20 -\%20L'analyse $\% 20 \mathrm{de} \% 20$ similitude $\% 20$ appliquee $\% 20$ aux $\% 20$ corpus $\% 20$ textuels.pdf

10. Camargo BV, Ana MJ. IRAMUTEQ: un software libre para el análisis de datos textuales. Temas Psicol. 2013; 21(2):513-8. doi: http://dx.doi. org/10.9788/TP2013.2-16

11. Salla F. Vigiar e punir e os estudos prisionais no Brasil. DILEMAS Rev Estud Conflito Control Soc [internet]. 2017 [citado 2019 jul. 25]; (2):29-43. Disponível em: https://revistas.ufrj.br/index. php/dilemas/article/download/14201/9534

12. Maffacciolli R, Oliveira DLLC. Challenges and perspectives of nursing care to vulnerable populations. Rev Gaúcha Enferm. 2018; 39:e20170189. doi: dx.doi.org/10.1590/1983-1447.2018.20170189

13. Zackseski C, Machado BA, Azevedo G. Dimensões do encarceramento e desafios da política penitenciária no Brasil. Rev Bras Ciênc Criminais [Internet]. 2016 [citado 2019 mai 15]; 126:291-331. Disponível em: http://www.mpsp.mp.br/portal/ page/portal/documentacao_e_divulgacao/doc biblioteca/bibli_servicos_produtos/bibli_boletim/bibli_bol_2006/RBCCrim_n.126.10.PDF

14. Lopes ALM, Fracolli LA. Revisão sistemática de literatura e metassíntese qualitativa: considerações sobre sua aplicação na pesquisa em enfermagem. Texto Contexto Enferm. 2008; 17(4):771-8. doi: dx.doi.org/10.1590/S0104-07072008000400020

15. Taquett SR, Maria CM. Analysis of qualitative studies conducted by physicians and published in Brazilian scientific journals between 2004 and 2013. Physis Rev Saúde Coletiva. 2016; 26(2):417-34. doi: http://dx.doi.org/10.1590/ S0103-73312016000200005

16. Ferreira MA. Theory of Social Representations and contributions to the research of health care and nursing. Esc Anna Nery. 2016; 20(2):214-5. doi: http://dx.doi.org/10.5935/1414-8145.20160028

17. Mcwen M, Wills EM. Bases teóricas da enfermagem. Porto Alegre: Artmed; 2016.

18. Santos MV, Alves VH, Pereira AV, Rodrigues DP, Marchiori GRS, Guerra JVV. The physical health of women deprived of their freedom in a prison in the state of Rio de Janeiro. Esc Anna Nery. 2017; doi: dx.doi.org/10.5935/1414-8145.20170033

19. Souza GC, Cabral KDS, Salgueiro CDBL. Reflexões sobre a assistência em enfermagem à mulher encarcerada: um estudo de revisão integrativa. Arq Ciênc Saúde UNIPAR; 2018. 22(1):55-62. doi: dx.doi.org/10.25110/arqsaude.v22i1.2018.6240

20. Barros MAR, Cavalcanti SDC, Galiza DF, Machado LG. Sociodemographic and reproductive factors of female prisoners. Rev Pesqui Cuidad Fundam on line 2016; 8(4):4980-84. doi: http://dx.doi. org/10.9789/2175-5361.2016.v8i4.49804985

21. Ministério da Saúde (BR). Política Nacional de Atenção Integral à Saúde das Pessoas Privadas de Liberdade no Sistema Prisional (PNAISP). Brasília: Ministério da Saúde; 2014.

22. Barbosa ML, Celino SDM, Pedraza DF, Costa GMC. Primary health care of convicts in the penitentiary system: subsides for nursing performance. Esc Anna Nery. 2014; 18(4):586-92 doi: http://dx.doi. org/10.5935/1414-8145.20140083

23. Santos MV, Alves VH, Pereira AV, Rodrigues DP, Marchiori GRS, Guerra JVV. The physical health of women deprived of their freedom in a prison in the state of Rio de Janeiro. Esc Anna Nery. 2017; 21(2):e20170033. doi: http://dx.doi. org/10.5935/1414-8145.20170033

24. Fernandes MA, Bezerra MM, Moura FMJSP, Alencar NES, Lima FFF, Castro AED. Sexually transmitted infections and the experiences of women in situations of imprisonment. Rev Enferm UERJ. 2016; 24(6):1-6. doi: http://dx.doi.org/10.12957/reuerj.2016.27774 
25. Diuana V, Ventura M, Silmas L, Larouzé B, Correa M. Women's reproductive rights in the penitentiary system: tensions and challenges in the transformation of reality. Ciênc Saúde Coletiva. 2016; 21(7):2041-50. doi: http://dx.doi. org/10.1590/1413-81232015217.21632015

26. Oliveira LV, Miranda FAN, Costa GMC. Women's reproductive rights in the penitentiary system: tensions and challenges in the transformation of reality. Rev Eletr Enferm. 2015; 17(2):360-9. doi: http://dx.doi.org/10.5216/ree.v17i2.29784

27. Ghidei L, Ramos SZ, Brousseau EC, Clarke JG, Mphprison: pipeline to women's preventative health. RI Med J [Internet]. 2018 [cited set 02, 2019]; 101:23-2. Available from: https://www.ncbi.nlm. nih.gov/pmc/articles/PMC6546428
28. Félix RS, França DJR, Nunes JT, Cunha ICBC, Davim RMB, Pereira JB. The nurse in pre-natal care for women in prison system. Rev Enferm UFPE on line [Internet]. 2017 [cited set 02, 2019]; 11(10):393647. Available from: https://periodicos.ufpe.br/ revistas/revistaenfermagem/article/view/15187

29. Ventura M, Simas L, Larouzpe B. Motherhood behind bars: the struggle for citizens' rights and health for women inmates and their children in Brazil. Cad Saúde Pública. 2015; 31(3):607-19. doi: dx.doi.org/10.1590/0102-311x00092914 